\title{
Einstein-Podolsky-Rosen correlations and Galilean transformations
}

\author{
Paweł Caban 7 Jakub Rembieliński团 Kordian A. Smoliński团 and Zbigniew Walczak \\ Department of Theoretical Physics, University of Łódź, ul. Pomorska 149/153, 90-236 Łódź, Poland
}

(Dated: 30 September 2002)

\begin{abstract}
In this paper we calculate with full details Einstein-Podolsky-Rosen spin correlations in the framework of nonrelativistic quantum mechanics. We consider the following situation: two-particle state is prepared (we consider separately distinguishable and identical particles and take into account the space part of the wave function) and two observers in relative motion measure the spin component of the particle along given directions. The measurements are performed in bounded regions of space (detectors), not necessarily simultaneously. The resulting correlation function depends not only on the directions of spin measurements but also on the relative velocity of the observers.
\end{abstract}

PACS numbers: 03.65.Ud

\section{INTRODUCTION}

The issue of locality plays a central role in recent theoretical and experimental investigations of basic properties of quantum mechanics. The history of this long-standing problem began in 1935, when Einstein, Podolsky, and Rosen (EPR) published their paper [1]. EPR considered a gedanken experiment with two spatially separated particles, $a$ and $b$, in an entangled state in which the relative position, $x_{a}-x_{b}$, and the total momentum, $p_{a}+p_{b}$, have definite values. If the momentum of the particle $a$ is measured one can predict with certainty the momentum of the particle $b$. Since particles are spatially separated and the locality of quantum mechanics is assumed, the measurement on the particle $a$ does not disturb the particle $b$, thus, due to the EPR reality criterion, the momentum of the particle $b$ is an element of reality. Alternatively one could measure the position of the particle $a$, and by the same arguments one concludes that also the position of the particle $b$ is an element of reality. But quantum mechanics does not allow us to find simultaneously values of $p_{b}$ and $x_{b}$, therefore EPR concluded that the description of reality that is provided by quantum mechanics is not complete.

In the above so-called "EPR paradox," the Einstein locality principle, which states "... the real factual situation of the system S2 is independent on what is done with the system S1, which is spatially separated from the former," [2] was applied for the first time explicitly to quantum mechanics.

For a long time, EPR predictions were experimentaly untestable. The problem was reformulated in terms of spin variables by Bohm [3], and in 1964 Bell proved that [4] in such a setting some inequality should hold for any local realistic theory. The Bell inequality was easier to handle experimentally because it imposes some constraints only on correlations of results of measurements performed by two distant observers. Many experiments were performed to test Bell-type inequalities [5, 6, 7, 8, 9, 10, 11], and all of them showed that

\footnotetext{
*Electronic address: P.Caban@merlin.fic.uni.lodz.pl

${ }^{\dagger}$ Electronic address: J.Rembielinski@merlin.fic.uni.lodz.pl

${ }^{\ddagger}$ Electronic address: K.A.Smolinski@merlin.fic.uni.lodz.pl

§Electronic address:Z.Walczak@merlin.fic.uni.lodz.pl
}

they are violated and that quantum-mechanical predictions are satisfied [23]. Recently, even experiments with observers in relative motion were performed [12].

In the standard formulation of the Bell inequality, only the spin part of the wave function of two particles is taken into account [13, 14]. However, some authors pointed out that when the issue of locality in quantum mechanics is considered, the space part of the wave function cannot be neglected [15, 16]. We accept this point of view. Unfortunately, in the standard formulation of relativistic quantum mechanics, the notion of localization of a particle is ill-defined. The main problem one encounters in this case concerns the Lorentz covariance of the localization (see, e.g., [17]). It causes the framework of the standard relativistic quantum mechanics to be unsuitable for the calculation of EPR correlations in the most general case, i.e., when the space part of the wave function and the relative motion of the observers are taken into account. In the framework of standard relativistic quantum mechanics, the spin correlations were calculated in [18, 19] but the derivation of the correlation function presented therein does not involve localization of measured particles in detectors and is restricted to the measurements performed in the same inertial frame. The framework of the Lorentz covariant quantum mechanics developed in [20] seems to be more suitable to calculate the EPR correlation function in the general case. The correlation function in such a framework was calculated in [21].

On the other hand, to the best of our knowledge,no systematic review of EPR correlations in the framework of nonrelativistic quantum mechanics in the general case exist in the literature. One of the reasons for this is that the EPR paradox appears only in the relativistic case. But in our opinion, we should know also the exact form of the EPR correlation function in the case of nonrelativistic quantum mechanics, at least to compare it with results obtained in the relativistic case. Therefore, the main goal of our present paper is to calculate in detail spin correlations in the framework of nonrelativistic quantum mechanics. More precisely, we consider the following situation: a two-particle state is prepared (we consider separately distinguishable and identical particles and we take into account the space part of the wave function) and two observers in relative motion measure the spin component of the particle along given directions. The measurements are performed in bounded regions of space (detectors) not necessar- 
ily simultaneously.

The paper is organized as follows. In Sec. II we calculate EPR spin correlations taking into account the space part of the wave function and motion of the observers. We discuss the cases of distinguishable and identical particles separately. Section [II concludes with a summary of our results. The main facts concerning the Galilean group and its unitary ray representations are collected in the Appendix.

\section{EPR CORRELATIONS}

In this section we calculate quantum correlations in the following case. In a given inertial frame of reference $\mathcal{O}$, a twoparticle state is prepared. Two observers, say $\mathcal{A}$ and $\mathcal{B}$, travel with constant velocities with respect to the frame $\mathcal{O}$. Each observer possesses a detector which can measure the spin component of a particle along a given axis fixed by unit vectors $\mathbf{a}$ and $\mathbf{b}$, respectively. We assume that the spin measurements take place only if the particle is inside the detector. Thus we assume that detectors occupy regions $A$ and $B$, respectively. We consider separately the case of distinguishable and identical particles. For the notation concerning the Galilean group and its unitary ray representations, see the Appendix.

\section{A. Distinguishable particles}

We consider two spin $s$ particles, say $\alpha$ and $\beta$. We assume that spins of both particles are equal for simplicity, however it is straightforward to generalize our considerations for the case of particles with different spins. The space of states of this two-particle system is $\mathcal{H}^{\alpha} \otimes \mathcal{H}^{\beta}$, where $\mathcal{H}^{\alpha}$ and $\mathcal{H}^{\beta}$ denote the space of states of the particles $\alpha$ and $\beta$, respectively. In the spaces $\mathcal{H}^{\alpha}$ and $\mathcal{H}^{\beta}$, we will use bases $\left\{\left|\mathbf{x}_{\alpha}, \mathbf{n}_{\alpha}, \lambda_{\alpha}\right\rangle\right\}$ and $\left\{\left|\mathbf{x}_{\beta}, \mathbf{n}_{\beta}, \lambda_{\beta}\right\rangle\right\}$, respectively. A vector $\left|\mathbf{x}_{\alpha}, \mathbf{n}_{\alpha}, \lambda_{\alpha}\right\rangle\left(\left|\mathbf{x}_{\beta}, \mathbf{n}_{\beta}, \lambda_{\beta}\right\rangle\right)$ describes the situation in which the particle $\alpha(\beta)$ is localized at $\mathbf{x}_{\alpha}\left(\mathbf{x}_{\beta}\right)$ and its spin component along the direction determined by a unit vector $\mathbf{n}_{\alpha}\left(\mathbf{n}_{\beta}\right)$ is equal to $\lambda_{\alpha}\left(\lambda_{\beta}\right)$. Definition of the vectors $|\mathbf{x}, \mathbf{n}, \lambda\rangle$ and their basic properties are given in the Appendix, Eqs. A.24 and A.25. We want to describe an EPR-type experiment in which two distant observers $\mathcal{A}$ and $\mathcal{B}$ measure spin components of the particles using detectors that occupy some bounded regions $A$ and $B$, respectively. Thus the measurement consists of the localization inside the region of the detector and simultaneous measurement of the spin component. Therefore, corresponding observables for particles $\alpha$ and $\beta$ read

$$
\Lambda_{A, \mathbf{a}}^{s} \otimes I, \quad I \otimes \Lambda_{B, \mathbf{b}}^{s}
$$

where the spectral decomposition of $\Lambda_{\Omega, \mathbf{n}}^{s}$ is the following:

$$
\Lambda_{\Omega, \mathbf{n}}^{s}=\sum_{\lambda=-s}^{s} \lambda\left(\int_{\Omega} d^{3} \mathbf{x}|\mathbf{x}, \mathbf{n}, \lambda\rangle\langle\mathbf{x}, \mathbf{n}, \lambda|\right) \equiv \sum_{\lambda=-s}^{s} \lambda \Pi_{\Omega, \mathbf{n}}^{s, \lambda} .
$$

The projectors $\Pi_{\Omega, \mathbf{n}}^{s, \lambda}$ in Eq. (2) have the following obvious interpretation: When we measure $\Pi_{\Omega, \mathbf{n}}^{s, \lambda}$ we get the value 1 if and only if the corresponding particle is inside $\Omega$ and its spin component along the direction $\mathbf{n}$ is equal to $\lambda$.

Under Galilean boosts, projector $\prod_{\Omega, \mathbf{n}}^{s, \lambda}$ transforms as follows [cf. Eq. A.34]:

$$
\begin{aligned}
U_{t}^{\dagger}(\mathbf{v}) \Pi_{\Omega, \mathbf{n}}^{s, \lambda} U_{t}(\mathbf{v}) & =\int_{\Omega} d^{3} \mathbf{x}|\mathbf{x}-t \mathbf{v}, \mathbf{n}, \lambda\rangle\langle\mathbf{x}-t \mathbf{v}, \mathbf{n}, \lambda| \\
& =\int_{\Omega^{\prime}(t)} d^{3} \mathbf{x}|\mathbf{x}, \mathbf{n}, \lambda\rangle\langle\mathbf{x}, \mathbf{n}, \lambda| \\
& =\Pi_{\Omega^{\prime}(t), \mathbf{n}}^{s, \lambda}
\end{aligned}
$$

where $\Omega^{\prime}(t)=\left\{\mathbf{x}^{\prime}: \mathbf{x}^{\prime}=\mathbf{x}-\mathbf{v} t, \mathbf{x} \in \Omega\right\}$. This means that localization in nonrelativistic quantum mechanics is covariant; that is, the projector $\Pi_{\Omega^{\prime}(t), \mathbf{n}}^{s, \lambda}$ corresponds to the localization in the same region as seen by the moving observer, at the moment $t$. We point out that this is not true in standard relativistic quantum mechanics [17].

Now we can calculate quantum correlations. This can be done in the following steps.

(i) Preparation of the initial state. We assume that a twoparticle state $\rho$ is prepared in a certain inertial frame of reference $\mathcal{O}$. Two other inertial frames of reference, $\mathcal{A}$ and $\mathcal{B}$, move with constant velocities with respect to $\mathcal{O}$. We denote the velocity of the frame $\mathcal{O}$ with respect to $\mathcal{A}$ and $\mathcal{B}$ by $\mathbf{v}_{A}$ and $\mathbf{v}_{B}$, respectively.

(ii) Measurement performed by observer $\mathcal{A}$. An observer at rest with respect to $\mathcal{A}$ (for simplicity, we refer to him as to the observer $\mathcal{A}$ ) measures at time $t_{A}$ the observable $\Lambda_{A, \mathbf{a}}^{s} \otimes I$. As a result of the measurement with selection, $\mathcal{A}$ receives a value $\lambda_{\alpha}$.

(iii) Free time evolution of the state. Next, the state evolves freely in time from $t_{A}$ to $t_{B} \geqslant t_{A}$.

(iv) Measurement performed by observer $\mathcal{B}$. At time $t_{B}$, an observer that rests with respect to $\mathcal{B}$ (we call him observer $\mathcal{B}$ ) measures $I \otimes \Lambda_{B, \mathbf{b}}^{s}$. The result of this measurement with selection is $\lambda_{\beta}$.

Let us denote the probability that $\mathcal{A}$ receives $\lambda_{\alpha}$ and $\mathcal{B}$ receives $\lambda_{\beta}$ as $p\left(\lambda_{\alpha}, \lambda_{\beta}\right)$. In the case of distinguishable particles, we define the following correlation function:

$$
\mathcal{C}^{\alpha, \beta}(\mathbf{a}, \mathbf{b})=\sum_{\lambda_{\alpha}, \lambda_{\beta}} \lambda_{\alpha} \lambda_{\beta} p\left(\lambda_{\alpha}, \lambda_{\beta}\right) .
$$

We could also imagine the situation in which the observers do not distingiush the type of particles. In such a case, the correlation function (4) should be replaced by

$$
\begin{aligned}
\mathcal{C}(\mathbf{a}, \mathbf{b}) & =\sum_{\lambda_{\alpha}, \lambda_{\beta}} \lambda_{\alpha} \lambda_{\beta}\left[p\left(\lambda_{\beta}, \lambda_{\alpha}\right)+p\left(\lambda_{\alpha}, \lambda_{\beta}\right)\right] \\
& =\mathcal{C}^{\alpha, \beta}(\mathbf{a}, \mathbf{b})+\mathcal{C}^{\beta, \alpha}(\mathbf{a}, \mathbf{b}) .
\end{aligned}
$$

Thus we have to calculate $p\left(\lambda_{\alpha}, \lambda_{\beta}\right)$. We do it according to the steps described above. 
(i) Preparation of the initial state. An initial state is prepared in the frame $\mathcal{O}$. At time, $t_{A}$ it is given by $\rho\left(t_{A}\right)$.

(ii) Measurement performed by the observer $\mathcal{A}$. For the observer $\mathcal{A}$, the density matrix $\rho\left(t_{A}\right)$ has the form

$$
\rho_{\mathcal{A}}\left(t_{A}\right)=U_{t_{A}}\left(\mathbf{v}_{A}\right) \rho\left(t_{A}\right) U_{t_{A}}^{\dagger}\left(\mathbf{v}_{A}\right)
$$

where $U_{t}(\mathbf{v})=U_{t}^{\alpha}(\mathbf{v}) \otimes U_{t}^{\beta}(\mathbf{v})$ and the unitary operator of pure Galilean boost $U_{t}^{\alpha}(\mathbf{v})\left[U_{t}^{\beta}(\mathbf{v})\right]$ is given in the Appendix; see Eqs. A.12, A.15, A.23, and A.34. Now the observer $\mathcal{A}$ measures $\Lambda_{A, \mathbf{a}}^{s} \otimes I$ in the state (6) and as a result of the measurement with selection he receives $\lambda_{\alpha}$ with the probability

$$
p\left(\lambda_{\alpha}\right)=\operatorname{Tr}\left[\rho_{\mathcal{A}}\left(t_{A}\right)\left(\Pi_{A, \mathbf{a}}^{s, \lambda_{\alpha}} \otimes I\right)\right] .
$$

The measurement reduces the density matrix (6) to

$$
\rho_{\mathcal{A}}^{\lambda_{\alpha}}\left(t_{A}\right)=\frac{\left(\Pi_{A, \mathbf{a}}^{s, \lambda_{\alpha}} \otimes I\right) \rho_{\mathcal{A}}\left(t_{A}\right)\left(\Pi_{A, \mathbf{a}}^{s, \lambda_{\alpha}} \otimes I\right)}{\operatorname{Tr}\left[\rho_{\mathcal{A}}\left(t_{A}\right)\left(\Pi_{A, \mathbf{a}}^{s, \lambda_{\alpha}} \otimes I\right)\right]} .
$$

(iii) Free time evolution of the state. The density matrix (8) as seen from the frame $\mathcal{O}$ reads

$$
\rho^{\lambda_{\alpha}}\left(t_{A}\right)=U_{t_{A}}^{\dagger}\left(\mathbf{v}_{A}\right) \rho_{\mathcal{A}}^{\lambda_{\alpha}}\left(t_{A}\right) U_{t_{A}}\left(\mathbf{v}_{A}\right)
$$

Now the state $\rho^{\lambda_{\alpha}}\left(t_{A}\right)$ evolves from time $t_{A}$ to $t_{B}$ and the resulting density matrix reads

$$
\rho^{\lambda_{\alpha}}\left(t_{B}\right)=U^{\dagger}\left(t_{B}-t_{A}\right) \rho^{\lambda_{\alpha}}\left(t_{A}\right) U\left(t_{B}-t_{A}\right)
$$

where $U\left(t_{B}-t_{A}\right)=U^{\alpha}\left(t_{B}-t_{A}\right) \otimes U^{\beta}\left(t_{B}-t_{A}\right)$ and $U(t)$ denotes the time evolution operator.

(iv) Measurement performed by the observer $\mathcal{B}$. The density matrix (10) as seen by the observer $\mathcal{B}$ has the form

$$
\rho_{\mathcal{B}}^{\lambda_{\alpha}}\left(t_{B}\right)=U_{t_{B}}\left(\mathbf{v}_{B}\right) \rho^{\lambda_{\alpha}}\left(t_{B}\right) U_{t_{B}}^{\dagger}\left(\mathbf{v}_{B}\right)
$$

Now the observer $\mathcal{B}$ measures $I \otimes \Lambda_{B, \mathbf{b}}^{s}$ in the state (11) and receives $\lambda_{\beta}$ with the probability

$$
p\left(\lambda_{\beta} \mid \lambda_{\alpha}\right)=\operatorname{Tr}\left[\rho_{\mathcal{B}}^{\lambda_{\alpha}}\left(t_{B}\right)\left(I \otimes \Pi_{B, \mathbf{b}}^{s, \lambda_{\beta}}\right)\right]
$$

It is conditional probability because the state in which $\mathcal{B}$ performs the measurement has the form 10 only if $\mathcal{A}$ receives $\lambda_{\alpha}$ in the first measurement.

So finally we get

$$
p\left(\lambda_{\alpha}, \lambda_{\beta}\right)=p\left(\lambda_{\alpha}\right) p\left(\lambda_{\beta} \mid \lambda_{\alpha}\right)
$$

Taking into account Eqs. (6) - 12, we can write

$$
\begin{aligned}
p\left(\lambda_{\alpha}, \lambda_{\beta}\right)= & \operatorname{Tr}\left\{\left[U_{t_{A}}^{\dagger}\left(\mathbf{v}_{A}\right)\left(\Pi_{A, \mathbf{a}}^{s, \lambda_{\alpha}} \otimes I\right) U_{t_{A}}\left(\mathbf{v}_{A}\right)\right] \rho\left(t_{A}\right)\left[U_{t_{A}}^{\dagger}\left(\mathbf{v}_{A}\right)\left(\Pi_{A, \mathbf{a}}^{s, \lambda_{\alpha}} \otimes I\right) U_{t_{A}}\left(\mathbf{v}_{A}\right)\right]\right. \\
& \left.\times\left[U\left(t_{B}-t_{A}\right) U_{t_{B}}^{\dagger}\left(\mathbf{v}_{B}\right)\left(I \otimes \Pi_{B, \mathbf{b}}^{s, \lambda_{\beta}}\right) U_{t_{B}}\left(\mathbf{v}_{B}\right) U^{\dagger}\left(t_{B}-t_{A}\right)\right]\right\} .
\end{aligned}
$$

Inserting Eq. (14) into Eq. (4), we get

$$
\mathcal{C}^{\alpha \beta}(\mathbf{a}, \mathbf{b})=\operatorname{Tr}\left\{\rho\left(t_{A}\right)\left[U_{t_{A}}^{\alpha \dagger}\left(\mathbf{v}_{A}\right) \Lambda_{A, \mathbf{a}}^{s} U_{t_{A}}^{\alpha}\left(\mathbf{v}_{A}\right)\right] \otimes\left[U^{\beta}\left(t_{B}-t_{A}\right) U_{t_{B}}^{\beta \dagger}\left(\mathbf{v}_{B}\right) \Lambda_{B, \mathbf{b}}^{s} U_{t_{B}}^{\beta}\left(\mathbf{v}_{B}\right) U^{\beta \dagger}\left(t_{B}-t_{A}\right)\right]\right\} .
$$

The function $\mathcal{C}^{\beta \alpha}(\mathbf{a}, \mathbf{b})$ can be obtained from $\mathcal{C}^{\alpha \beta}(\mathbf{a}, \mathbf{b})$ by simultaneous change of the order in the tensor product and change of indices $\alpha$ and $\beta$.

The formula (15) may be simplified in a particular case when the initial state is a pure one. In this case $\rho\left(t_{A}\right)=|\psi\rangle\langle\psi|$, where $|\psi\rangle \in \mathcal{H}^{\alpha} \otimes \mathcal{H}^{\beta}$ is normalized.

Moreover, one can check that in the free time evolution case, the following relation holds:

$$
U(\tau)\left[U_{t}^{\dagger}(\mathbf{v}) \Pi_{\Omega, \mathbf{n}}^{s, \lambda} U_{t}(\mathbf{v})\right] U^{\dagger}(\tau)=\frac{1}{(2 \pi)^{3}} \iint d^{3} \mathbf{k} d^{3} \mathbf{p} \int_{\Omega} d^{3} \mathbf{x} e^{i(\mathbf{x}-t \mathbf{v}) \cdot(\mathbf{p}-\mathbf{k})+\left[i \tau\left(\mathbf{k}^{2}-\mathbf{p}^{2}\right) / 2 M\right]}|\mathbf{k}, \mathbf{n}, \lambda\rangle\langle\mathbf{p}, \mathbf{n}, \lambda| .
$$

Thus inserting Eq. (3) and (16) into Eq. (15), we have

$$
\begin{aligned}
\mathcal{C}_{\psi}^{\alpha \beta}(\mathbf{a}, \mathbf{b})= & \frac{1}{(2 \pi)^{3}} \int_{A} d^{3} \mathbf{x} \int_{B} d^{3} \mathbf{y} \iint d^{3} \mathbf{k} d^{3} \mathbf{p}\left\{e^{i\left(\mathbf{y}-\mathbf{v}_{B} t_{B}\right) \cdot(\mathbf{p}-\mathbf{k})+\left[i\left(t_{B}-t_{A}\right) / 2 M_{\beta}\right]\left(\mathbf{k}^{2}-\mathbf{p}^{2}\right)}\right. \\
& \left.\times \sum_{\lambda_{\alpha}, \lambda_{\beta}} \lambda_{\alpha} \lambda_{\beta}\left\langle\psi\left|\left[\left|\mathbf{x}-\mathbf{v}_{A} t_{A}, \mathbf{a}, \lambda_{\alpha}\right\rangle\left\langle\mathbf{x}-\mathbf{v}_{A} t_{A}, \mathbf{a}, \lambda_{\alpha}|\otimes| \mathbf{k}, \mathbf{b}, \lambda_{\beta}\right\rangle\left\langle\mathbf{p}, \mathbf{b}, \lambda_{\beta}\right|\right]\right| \psi\right\rangle\right\},
\end{aligned}
$$

where $M_{\beta}$ denotes the mass of the particle $\beta$. In the position representation, $|\psi\rangle$ is of the form

$$
|\psi\rangle=\sum_{m_{\alpha}, m_{\beta}} \iint d^{3} \mathbf{x} d^{3} \mathbf{y} \psi_{m_{\alpha} m_{\beta}}(\mathbf{x}, \mathbf{y})\left|\mathbf{x}, m_{\alpha}\right\rangle \otimes\left|\mathbf{y}, m_{\beta}\right\rangle
$$


Note that in Eq. (18) we use the basis $\left\{\left|\mathbf{x}, m_{\alpha}\right\rangle \otimes\left|\mathbf{y}, m_{\beta}\right\rangle\right\}$, where $m_{\alpha}$ and $m_{\beta}$ denote spin components along the $z$ axis. One can check that under the definition (18) and using Eq. A.30 and A.31 we obtain

$$
\begin{gathered}
\mathcal{C}_{\psi}^{\alpha \beta}(\mathbf{a}, \mathbf{b})=\frac{1}{(2 \pi)^{6}} \int_{A} d^{3} \mathbf{x} \int_{B} d^{3} \mathbf{y} \iint d^{3} \mathbf{k} d^{3} \mathbf{p} e^{i\left(\mathbf{y}-\mathbf{v}_{B} t_{B}\right) \cdot(\mathbf{p}-\mathbf{k})+\left[i\left(t_{B}-t_{A}\right) / 2 M_{B}\right]\left(\mathbf{k}^{2}-\mathbf{p}^{2}\right)} \sum_{\substack{m_{\alpha}, m_{\beta} \\
m_{\alpha}^{\prime}, m_{\beta}^{\prime}}} \iint d^{3} \mathbf{x}^{\prime} d^{3} \mathbf{y}^{\prime} e^{i\left(\mathbf{k} \cdot \mathbf{x}^{\prime}-\mathbf{p} \cdot \mathbf{y}^{\prime}\right)} \\
\times \psi_{m_{\alpha}^{\prime} m_{\beta}^{\prime}}^{\star}\left(\mathbf{x}-\mathbf{v}_{A} t_{A}, \mathbf{x}^{\prime}\right) \psi_{m_{\alpha} m_{\beta}}\left(\mathbf{x}-\mathbf{v}_{A} t_{A}, \mathbf{y}^{\prime}\right)(\mathbf{a} \cdot \mathbf{S})_{m_{\alpha} m_{\alpha}^{\prime}}(\mathbf{b} \cdot \mathbf{S})_{m_{\beta} m_{\beta}^{\prime}} .
\end{gathered}
$$

When $t_{A}=t_{B}=t$ the correlation function 19 takes the form

$$
\mathcal{C}_{\psi}^{\alpha \beta}(\mathbf{a}, \mathbf{b})=\int_{A} d^{3} \mathbf{x} \int_{B} d^{3} \mathbf{y} \sum_{\substack{m_{\alpha}, m_{\beta} \\ m_{\alpha}^{\prime}, m_{\beta}^{\prime}}} \psi_{m_{\alpha}^{\prime} m_{\beta}^{\prime}}^{\star}\left(\mathbf{x}-\mathbf{v}_{A} t, \mathbf{y}-\mathbf{v}_{B} t\right) \psi_{m_{\alpha} m_{\beta}}\left(\mathbf{x}-\mathbf{v}_{A} t, \mathbf{y}-\mathbf{v}_{B} t\right)(\mathbf{a} \cdot \mathbf{S})_{m_{\alpha} m_{\alpha}^{\prime}}(\mathbf{b} \cdot \mathbf{S})_{m_{\beta} m_{\beta}^{\prime}}
$$

\section{The case $s=\frac{1}{2}$}

Now let us apply the formula (20) to the case of the lowest nontrivial spin $s=\frac{1}{2}$. The state $|\psi\rangle$ can be a triplet or a singlet. Singlet state. For the singlet state we have

$$
\psi_{m_{\alpha} m_{\beta}}(\mathbf{x}, \mathbf{y})=-\psi_{m_{\beta} m_{\alpha}}(\mathbf{x}, \mathbf{y})
$$

and then from Eq. (20) we receive

$$
\mathcal{C}_{\psi_{\text {singlet }}}^{\alpha \beta}(\mathbf{a}, \mathbf{b})=-\frac{1}{2} \cos \left(\theta_{a b}\right) \int_{A} d^{3} \mathbf{x} \int_{B} d^{3} \mathbf{y}\left|\psi_{\text {singlet }}\left(\mathbf{x}-\mathbf{v}_{A} t, \mathbf{y}-\mathbf{v}_{B} t\right)\right|^{2},
$$

where $\psi_{\text {singlet }}(\mathbf{x}, \mathbf{y}) \equiv \psi_{1 / 2,-1 / 2}(\mathbf{x}, \mathbf{y}), \theta_{a b}$ denotes an angle between vectors $\mathbf{a}$ and $\mathbf{b}$, and the normalization yields

$$
\iint d^{3} \mathbf{x} d^{3} \mathbf{y}\left|\psi_{\text {singlet }}(\mathbf{x}, \mathbf{y})\right|^{2}=\frac{1}{2}
$$

Thus the correlation function depends on the vectors $\mathbf{a}$ and $\mathbf{b}$ in the standard way, i.e., it behaves like a cosine of an angle between vectors $\mathbf{a}$ and $\mathbf{b}$. The only difference is the presence of the term $\int_{A} d^{3} \mathbf{x} \int_{B} d^{3} \mathbf{y}\left|\psi_{\text {singlet }}\left(\mathbf{x}-\mathbf{v}_{A} t, \mathbf{y}-\mathbf{v}_{B} t\right)\right|^{2}$, which influences the intensity of the correlations.

Triplet state. In the triplet case we have

$$
\psi_{m_{\alpha} m_{\beta}}(\mathbf{x}, \mathbf{y})=\psi_{m_{\beta} m_{\alpha}}(\mathbf{x}, \mathbf{y})
$$

and the correlation function has a rather complicated form,

$$
\begin{aligned}
\mathcal{C}_{\psi_{\text {triplet }} \beta}^{\alpha \beta}(\mathbf{a}, \mathbf{b})=\frac{1}{4} & \int_{A} d^{3} \mathbf{x} \int_{B} d^{3} \mathbf{y}\left\{\left(\left|\psi_{++}\right|^{2}+\left|\psi_{--}\right|^{2}\right) \cos \theta_{a} \cos \theta_{b}+\left(\psi_{++}^{\star} \psi_{--} e^{i\left(\varphi_{a}+\varphi_{b}\right)}+\psi_{--}^{\star} \psi_{++} e^{-i\left(\varphi_{a}+\varphi_{b}\right)}\right) \sin \theta_{a} \sin \theta_{b}\right. \\
& +\left(\psi_{++}^{\star} \psi_{+-}-\psi_{+-}^{\star} \psi_{--}\right)\left(\cos \theta_{a} \sin \theta_{b} e^{i \varphi_{b}}+\sin \theta_{a} \cos \theta_{b} e^{i \varphi_{a}}\right)+\left(\psi_{+-}^{\star} \psi_{++}-\psi_{--}^{\star} \psi_{+-}\right)\left(\cos \theta_{a} \sin \theta_{b} e^{-i \varphi_{b}}\right. \\
& \left.\left.+\sin \theta_{a} \cos \theta_{b} e^{-i \varphi_{a}}\right)-2 \psi_{+-}^{\star} \psi_{+-}\left[\cos \theta_{a} \cos \theta_{b}-\sin \theta_{a} \sin \theta_{b} \cos \left(\varphi_{a}-\varphi_{b}\right)\right]\right\}
\end{aligned}
$$

where we used the following notation:

$$
\begin{gathered}
\psi_{++} \equiv \psi_{1 / 2,1 / 2}\left(\mathbf{x}-\mathbf{v}_{A} t, \mathbf{y}-\mathbf{v}_{B} t\right) \\
\psi_{+-} \equiv \psi_{1 / 2,-1 / 2}\left(\mathbf{x}-\mathbf{v}_{A} t, \mathbf{y}-\mathbf{v}_{B} t\right) \\
\psi_{--} \equiv \psi_{-1 / 2,-1 / 2}\left(\mathbf{x}-\mathbf{v}_{A} t, \mathbf{y}-\mathbf{v}_{B} t\right) \\
\mathbf{a}=\left(\cos \varphi_{a} \sin \theta_{a}, \sin \varphi_{a} \sin \theta_{a}, \cos \theta_{a}\right), \\
\mathbf{b}=\left(\cos \varphi_{b} \sin \theta_{b}, \sin \varphi_{b} \sin \theta_{b}, \cos \theta_{b}\right),
\end{gathered}
$$

and the normalization yields

$$
\begin{array}{r}
\iint d^{3} \mathbf{x} d^{3} \mathbf{y}\left\{\left|\psi_{1 / 2,1 / 2}(\mathbf{x}, \mathbf{y})\right|^{2}+\left|\psi_{-1 / 2,-1 / 2}(\mathbf{x}, \mathbf{y})\right|^{2}+\right. \\
\left.2\left|\psi_{\frac{1}{2},-\frac{1}{2}}(\mathbf{x}, \mathbf{y})\right|^{2}\right\}=1 .
\end{array}
$$

The triplet correlation function given by Eq. 25 depends on velocities of frames in a more non-trivial way than in the singlet case. Moreover, it is evident that the dependence on frame 
velocities in fact reduces to the dependence on the relative velocity of the observers.

\section{B. Identical particles}

Now we calculate the correlation function in the same setting as in the previous subsection but in the case of two identical particles. We denote the one-particle Hilbert space by $\mathcal{H}$. For our purpose it is convenient to use in $\mathcal{H}$ the basis $\{|\mathbf{x}, \mathbf{n}, \lambda\rangle\}$, where, as previously, $|\mathbf{x}, \mathbf{n}, \lambda\rangle$ represents the particle localized at $\mathbf{x}$ and with spin component along the direction $\mathbf{n}$ equal to $\lambda$ [see also the Appendix, Eqs. A.24 and A.25]. Since the particles are identical, the state vectors of the twoparticle system constitute the symmetrical or antisymmetrical subspace of $\mathcal{H} \otimes \mathcal{H}$ and the observables are represented by symmetrical operators. Thus an observable that measures the spin component along direction $\mathbf{n}$ inside the region $\Omega$ has the form

$$
\Delta_{\Omega, \mathbf{n}}^{s}=\Lambda_{\Omega, \mathbf{n}}^{s} \otimes I+I \otimes \Lambda_{\Omega, \mathbf{n}}^{s}=\sum_{\lambda=-s}^{s} \lambda\left(\Pi_{\Omega, \mathbf{n}}^{s, \lambda} \otimes I+I \otimes \Pi_{\Omega, \mathbf{n}}^{s, \lambda}\right),
$$

where, as in the previous subsection,

$$
\begin{gathered}
\Pi_{\Omega, \mathbf{n}}^{s, \lambda} \equiv \int_{\Omega} d^{3} \mathbf{x}|\mathbf{x}, \mathbf{n}, \lambda\rangle\langle\mathbf{x}, \mathbf{n}, \lambda| \\
\Lambda_{\Omega, \mathbf{n}}^{s} \equiv \sum_{\lambda=-s}^{s} \lambda\left(\int_{\Omega} d^{3} \mathbf{x}|\mathbf{x}, \mathbf{n}, \lambda\rangle\langle\mathbf{x}, \mathbf{n}, \lambda|\right)=\sum_{\lambda=-s}^{s} \lambda \Pi_{\Omega, \mathbf{n}}^{s, \lambda}
\end{gathered}
$$

and $I$ denotes the identity. When we apply the observable (32), we do not know how many particles are inside $\Omega$. Thus $\Delta_{\Omega, \mathbf{n}}^{s}$ measures the component of the total spin of all particles inside $\Omega$. To justify the above statement, we consider the particle number operator $\Pi_{\Omega}^{s} \otimes I+I \otimes \Pi_{\Omega}^{s}$, where

$$
\Pi_{\Omega}^{s} \equiv \sum_{\lambda=-s}^{s} \int_{\Omega} d^{3} \mathbf{x}|\mathbf{x}, \mathbf{n}, \lambda\rangle\langle\mathbf{x}, \mathbf{n}, \lambda|=\sum_{\lambda=-s}^{s} \Pi_{\Omega, \mathbf{n}}^{s, \lambda}
$$

which discriminates how many particles are inside $\Omega$. The spectral decomposition of $\Pi_{\Omega}^{s} \otimes I+I \otimes \Pi_{\Omega}^{s}$ reads

$$
\Pi_{\Omega}^{s} \otimes I+I \otimes \Pi_{\Omega}^{s}=2 \Pi_{\Omega}^{(2)}+1 \Pi_{\Omega}^{(1)}+0 \Pi_{\Omega}^{(0)},
$$

where $\Pi_{\Omega}^{(2)}, \Pi_{\Omega}^{(1)}, \Pi_{\Omega}^{(0)}$ are projectors on mutually orthogonal subspaces and their explicit form is the following:

$$
\begin{gathered}
\Pi_{\Omega}^{(2)}=\Pi_{\Omega}^{s} \otimes \Pi_{\Omega}^{s}, \\
\Pi_{\Omega}^{(1)}=\Pi_{\Omega}^{s} \otimes I+I \otimes \Pi_{\Omega}^{s}-2 \Pi_{\Omega}^{s} \otimes \Pi_{\Omega}^{s}, \\
\Pi_{\Omega}^{(0)}=I \otimes I-\Pi_{\Omega}^{s} \otimes I-I \otimes \Pi_{\Omega}^{s}+\Pi_{\Omega}^{s} \otimes \Pi_{\Omega}^{s} .
\end{gathered}
$$

Therefore, as a result of measurement of $\Pi_{\Omega}^{s} \otimes I+I \otimes \Pi_{\Omega}^{s}$, we receive one of the following outcomes (i) there is no particle inside $\Omega$; (ii) there is one particle inside $\Omega$; and (iii) there are two particles inside $\Omega$.
In the sequel we will restrict ourselves to the simplest case $s=\frac{1}{2}$. Let us denote for simplicity

$$
\Pi_{\Omega, \mathbf{n}}^{+} \equiv \Pi_{\Omega, \mathbf{n}}^{1 / 2,1 / 2}, \quad \Pi_{\Omega, \mathbf{n}}^{-} \equiv \Pi_{\Omega, \mathbf{n}}^{1 / 2,-1 / 2} .
$$

The spectral decomposition of $\Delta_{\Omega, \mathbf{n}} \equiv \Delta_{\Omega, \mathbf{n}}^{1 / 2}$ has the form

$$
\begin{aligned}
\Delta_{\Omega, \mathbf{n}}=\frac{1}{2} \Pi_{\Omega, \mathbf{n}}^{(1,+)}-\frac{1}{2} \Pi_{\Omega, \mathbf{n}}^{(1,-)}+1 & \Pi_{\Omega, \mathbf{n}}^{(2,1)}-1 \Pi_{\Omega, \mathbf{n}}^{(2,-1)} \\
& +0 \Pi_{\Omega, \mathbf{n}}^{(2,0)}+0 \Pi_{\Omega}^{(0,0)},
\end{aligned}
$$

where

$$
\begin{gathered}
\Pi_{\Omega, \mathbf{n}}^{(1,+)}=\Pi_{\Omega, \mathbf{n}}^{+} \otimes I+I \otimes \Pi_{\Omega, \mathbf{n}}^{+}-2 \Pi_{\Omega, \mathbf{n}}^{+} \otimes \Pi_{\Omega, \mathbf{n}}^{+} \\
-\Pi_{\Omega, \mathbf{n}}^{+} \otimes \Pi_{\Omega, \mathbf{n}}^{-}-\Pi_{\Omega, \mathbf{n}}^{-} \otimes \Pi_{\Omega, \mathbf{n}}^{+} \\
\Pi_{\Omega, \mathbf{n}}^{(1,-)}=\Pi_{\Omega, \mathbf{n}}^{-} \otimes I+I \otimes \Pi_{\Omega, \mathbf{n}}^{-}-2 \Pi_{\Omega, \mathbf{n}}^{-} \otimes \Pi_{\Omega, \mathbf{n}}^{-} \\
-\Pi_{\Omega, \mathbf{n}}^{+} \otimes \Pi_{\Omega, \mathbf{n}}^{-}-\Pi_{\Omega, \mathbf{n}}^{-} \otimes \Pi_{\Omega, \mathbf{n}}^{+} \\
\Pi_{\Omega, \mathbf{n}}^{(2,1)}=\Pi_{\Omega, \mathbf{n}}^{+} \otimes \Pi_{\Omega, \mathbf{n}}^{+} \\
\Pi_{\Omega, \mathbf{n}}^{(2,-1)}=\Pi_{\Omega, \mathbf{n}}^{-} \otimes \Pi_{\Omega, \mathbf{n}}^{-} \\
\Pi_{\Omega, \mathbf{n}}^{(2,0)}=\Pi_{\Omega, \mathbf{n}}^{+} \otimes \Pi_{\Omega, \mathbf{n}}^{-}+\Pi_{\Omega, \mathbf{n}}^{-} \otimes \Pi_{\Omega, \mathbf{n}}^{+} \\
\Pi_{\Omega, \mathbf{n}}^{(0,0)}= \\
I \otimes I-\Pi_{\Omega, \mathbf{n}}^{+} \otimes I-\Pi_{\Omega, \mathbf{n}}^{-} \otimes I-I \otimes \Pi_{\Omega, \mathbf{n}}^{+}-I \otimes \Pi_{\Omega, \mathbf{n}}^{-} \\
+\Pi_{\Omega, \mathbf{n}}^{+} \otimes \Pi_{\Omega, \mathbf{n}}^{+}+\Pi_{\Omega, \mathbf{n}}^{-} \otimes \Pi_{\Omega, \mathbf{n}}^{-}+\Pi_{\Omega, \mathbf{n}}^{+} \otimes \Pi_{\Omega, \mathbf{n}}^{-} \\
+\Pi_{\Omega, \mathbf{n}}^{-} \otimes \Pi_{\Omega, \mathbf{n}}^{+}
\end{gathered}
$$

are projectors on mutually orthogonal subspaces and one can easily check that

$$
\begin{gathered}
\Pi_{\Omega, \mathbf{n}}^{(2)}=\Pi_{\Omega, \mathbf{n}}^{(2,1)}+\Pi_{\Omega, \mathbf{n}}^{(2,-1)}+\Pi_{\Omega, \mathbf{n}}^{(2,0)}, \\
\Pi_{\Omega, \mathbf{n}}^{(1)}=\Pi_{\Omega, \mathbf{n}}^{(1,+)}+\Pi_{\Omega, \mathbf{n}}^{(1,-)}, \\
\Pi_{\Omega, \mathbf{n}}^{(0)}=\Pi_{\Omega, \mathbf{n}}^{(0,0)}
\end{gathered}
$$

[cf. Eqs. (37)-39] ]. Thus we can see that the observable $\Delta_{\Omega, \mathbf{n}}$ really measures the component of the total spin of all the particles inside $\Omega$.

Now we are prepared to calculate quantum correlations in the case of two identical particles. As previously, we assume that a two-particle state $\rho\left(t_{A}\right)$ is prepared at time $t_{A}$ in a certain inertial frame of reference $\mathcal{O}$. Moreover, the two observers, $\mathcal{A}$ and $\mathcal{B}$, move with constant velocities with respect to $\mathcal{O}$. We denote the velocity of the frame $\mathcal{O}$ with respect to $\mathcal{A}$ and $\mathcal{B}$ by $\mathbf{v}_{A}$ and $\mathbf{v}_{B}$, respectively. The observer $\mathcal{A}$ measures at time $t_{A}$ the observable $\Delta_{A, \mathbf{a}}$, and as a result of measurement with selection he receives the value $\lambda$. Here $A$ denotes some bounded region in $\mathbb{R}^{3}$ and a denotes a unit vector. Next, at time $t_{B} \geqslant t_{A}$ the observer $\mathcal{B}$ measures $\Delta_{B, \mathbf{b}}$ and receives $\lambda^{\prime}$, where similarly $B \subset \mathbb{R}^{3}$ and $\mathbf{b}$ is a unit vector. We can write

$$
\begin{aligned}
& \Delta_{A, \mathbf{a}}=\sum_{N=0}^{2}\left(\sum_{\lambda_{N}} \lambda_{N} \Pi_{A, \mathbf{a}}^{\left(N, \lambda_{N}\right)}\right), \\
& \Delta_{B, \mathbf{b}}=\sum_{N=0}^{2}\left(\sum_{\lambda_{N}} \lambda_{N} \Pi_{B, \mathbf{b}}^{\left(N, \lambda_{N}\right)}\right) .
\end{aligned}
$$


For the explicit form of $\Pi_{A, \mathbf{a}}^{\left(N, \lambda_{N}\right)}$ and $\Pi_{B, \mathbf{b}}^{\left(N, \lambda_{N}\right)}$, see Eqs. (42)477. In Eqs. 51)-(52), $\lambda_{N}$ is an eigenvalue of $\Delta_{A, \mathbf{a}}, \Delta_{B, \mathbf{b}}$ and the projector $\Pi_{A, \mathbf{a}}^{\left(N, \lambda_{N}\right)}$ corresponds to the situation in which inside the region $A$ there are $N$ particles and the total spin component along the direction $\mathbf{a}$ of all these particles is equal to $\lambda_{N}$.

As in the previous section, let $p\left(\lambda, \lambda^{\prime}\right)$ denote the probability that $\mathcal{A}$ receives the value $\lambda$ and $\mathcal{B}$ the value $\lambda^{\prime}$. We define the correlation function by the formula

$$
\mathcal{C}(\mathbf{a}, \mathbf{b})=\sum_{\lambda, \lambda^{\prime}} \lambda \lambda^{\prime} p\left(\lambda, \lambda^{\prime}\right)
$$

This function differs from zero when each observer registers one particle or when one observer registers two particles and the second one registers one or two particles. If one of them registers no particle, then the corresponding $\lambda$ is equal to zero. We take into account only the case in which each observer registers one particle.

Performing similar steps as in the case of distinguishable particles and taking into account Eqs. 51) and (52), we get

$$
p\left(\lambda_{1}, \lambda_{1}^{\prime}\right)=\operatorname{Tr}\left\{\left[\mathcal{U}_{t_{A}}^{\dagger}\left(\mathbf{v}_{A}\right) \Pi_{A, \mathbf{a}}^{\left(1, \lambda_{1}\right)} \mathcal{U}_{t_{A}}\left(\mathbf{v}_{A}\right)\right] \rho\left(t_{A}\right)\left[\mathcal{U}_{t_{A}}^{\dagger}\left(\mathbf{v}_{A}\right) \Pi_{A, \mathbf{a}}^{\left(1, \lambda_{1}\right)} \mathcal{U}_{t_{A}}\left(\mathbf{v}_{A}\right)\right]\left[\mathcal{U}\left(t_{B}-t_{A}\right) \mathcal{U}_{t_{B}}^{\dagger}\left(\mathbf{v}_{B}\right) \Pi_{B, \mathbf{b}}^{\left(1, \lambda_{1}^{\prime}\right)} \mathcal{U}_{t_{B}}\left(\mathbf{v}_{B}\right) \mathcal{U}^{\dagger}\left(t_{B}-t_{A}\right)\right]\right\}
$$

where, as previously

$$
\mathcal{U}_{t}(\mathbf{v})=U_{t}(\mathbf{v}) \otimes U_{t}(\mathbf{v}), \quad \mathcal{U}(t)=U(t) \otimes U(t)
$$

and $U_{t}(\mathbf{v}), U(t)$ are defined in the Appendix; see Eqs. A.12, A.15, A.23, Eqs. A.34). Inserting Eq. 54) into Eq. (53), we arrive at the following formula for the correlation function:

$$
\begin{aligned}
\mathcal{C}(\mathbf{a}, \mathbf{b})= & \operatorname{Tr}\left\{\sum_{\lambda_{1}}\left[\mathcal{U}_{t_{A}}^{\dagger}\left(\mathbf{v}_{A}\right) \Pi_{A, \mathbf{a}}^{\left(1, \lambda_{1}\right)} \mathcal{U}_{t_{A}}\left(\mathbf{v}_{A}\right)\right] \rho\left(t_{A}\right)\left[\mathcal{U}_{t_{A}}^{\dagger}\left(\mathbf{v}_{A}\right) \Pi_{A, \mathbf{a}}^{\left(1, \lambda_{1}\right)} \mathcal{U}_{t_{A}}\left(\mathbf{v}_{A}\right)\right]\left[\mathcal{U}\left(t_{B}-t_{A}\right) \mathcal{U}_{t_{B}}^{\dagger}\left(\mathbf{v}_{B}\right) \Delta_{B, \mathbf{b}} \mathcal{U}_{t_{B}}\left(\mathbf{v}_{B}\right) \mathcal{U}^{\dagger}\left(t_{B}-t_{A}\right)\right]\right. \\
& \left.\times\left[\mathcal{U}_{t_{A}}^{\dagger}\left(\mathbf{v}_{A}\right) \Delta_{A, \mathbf{a}} \mathcal{U}_{t_{A}}\left(\mathbf{v}_{A}\right)\right]\right\} .
\end{aligned}
$$

Let us consider the simplest case in which both the observers rest with respect to $\mathcal{O}$ and the measurements are performed at the same time. It means that we put in Eq. (56)

$$
\mathbf{v}_{A}=\mathbf{0}, \quad \mathbf{v}_{B}=\mathbf{0}, \quad t_{A}=t_{B}=t .
$$

The result is the following

$$
\mathcal{C}(\mathbf{a}, \mathbf{b})=\sum_{\lambda_{1}} \operatorname{Tr}\left\{\Pi_{A, \mathbf{a}}^{\left(1, \lambda_{1}\right)} \rho(t) \Pi_{A, \mathbf{a}}^{\left(1, \lambda_{1}\right)} \Delta_{B, \mathbf{b}} \Delta_{A, \mathbf{a}}\right\} .
$$

Furthermore, if we assume that the regions $A$ and $B$ are disjoint (as it happens in the real EPR-type experiments) we find from (32) 33, 40, 42], and (43) that $\Delta_{B, \mathbf{b}}$ and $\Pi_{A, \mathbf{a}}^{\left(1, \lambda_{1}\right)}$ commute. Thus changing the order in Eq. (58) and using Eq. 51. we get

$$
\mathcal{C}(\mathbf{a}, \mathbf{b})=\operatorname{Tr}\left\{\rho(t) \Delta_{A, \mathbf{a}} \Delta_{B, \mathbf{b}}\right\}
$$

\section{CONCLUSIONS}

In this paper, we have presented the detailed calculation of the spin correlation functions in the EPR-type experiments. In opposition to the standard approach, we considered the space part of the wave function and the relative motion of the observers. We also took into account the fact that every measurement of the spin component is connected with the simultaneous localization of the particle inside the detector. Thus we assumed that observers perform measurements in some bounded regions of space. We performed our calculations in the framework of nonrelativistic quantum mechanics. There were two main reasons for this. First, to take into account the localization in the regions of the detectors, we need the welldefined notion of the localization (in the standard formulation of relativistic quantum mechanics, the notion of localization of the particles is ill-defined, however see [20]). Secondly, to the best of our knowledge, any systematic review of EPR correlations in the framework of nonrelativistic quantum mechanics in the general case mentioned above does not exist in the literature. Moreover, we considered separately the case of identical and distinguishable particles. In both cases, we found the general formula for the correlation function under the assumption that the initial two-particle state is arbitrary. In addition, we also considered some important special cases.

In the most interesting case of the singlet state of two spin$\frac{1}{2}$ particles, we determined, as one might expect, that the correlation function depends on the vectors $\mathbf{a}$ and $\mathbf{b}$ in the standard way, i.e., it behaves like a cosine of an angle between vectors $\mathbf{a}$ and $\mathbf{b}$. The only difference is the presence of 
the term $\int_{A} d^{3} \mathbf{x} \int_{B} d^{3} \mathbf{y}\left|\psi_{\text {singlet }}\left(\mathbf{x}-\mathbf{v}_{A} t, \mathbf{y}-\mathbf{v}_{B} t\right)\right|^{2}$, which influences the intensity of the correlations. Note that the low velocity limit of the relativistic correlation function for the singlet state of two spin- $\frac{1}{2}$ particles derived in [18, 19] and in [21] differs from our results. The correlation function obtained in [18, 19] depends on the state of motion of the particles, while the function derived in [21] contains a correction of second order in velocities to our nonrelativistic formula. However, in this second case, when both measurements are performed in the same inertial frame, the limiting correlation function coincides with our results.

\section{APPENDIX: GALILEAN GROUP}

To establish notation and conventions, we summarize here the main facts concerning the Galilean group and its unitary representations. Let $\mathcal{H}$ be the one-particle Hilbert space of states. In this space, the following basic observables exist: $\hat{\mathbf{X}}$ (position), $\hat{\mathbf{P}}$ (momentum), $\hat{\mathbf{S}}$ (spin). They fulfil the following relations:

$$
\begin{gathered}
{\left[\hat{X}_{i}, \hat{X}_{j}\right]=0, \quad\left[\hat{P}_{i}, \hat{P}_{j}\right]=0} \\
{\left[\hat{X}_{i}, \hat{P}_{j}\right]=i \delta_{i j}, \quad\left[\hat{X}_{i}, \hat{S}_{j}\right]=0} \\
{\left[\hat{P}_{i}, \hat{S}_{j}\right]=0, \quad\left[\hat{S}_{i}, \hat{S}_{j}\right]=i \varepsilon_{i j k} \hat{S}_{k}}
\end{gathered}
$$

The Galilean group and its algebra act in the space $\mathcal{H}$. As is well known, classical Galilean transformations have the following form

$$
\begin{gathered}
\mathbf{x}^{\prime}=R \mathbf{x}+\mathbf{a}-\mathbf{v} t \\
t^{\prime}=t+\tau,
\end{gathered}
$$

where $\mathbf{v}$ denotes the velocity of the frame $\left(\mathbf{x}^{\prime}, t^{\prime}\right)$ with respect to the frame $(\mathbf{x}, t)$ and we adopt the passive point of view. In the Hilbert, space the rotation $R$ is generated by the total angular momentum $\hat{\mathbf{J}}$, the translation a is generated by the momentum $\hat{\mathbf{P}}$, the time translation is generated by the Hamiltonian $\hat{H}$ and Galilean boost is generated by $\hat{\mathbf{G}}$. The basic commutation relations of the Galilean algebra (in fact its central extension) read

$$
\begin{gathered}
{\left[\hat{P}_{i}, \hat{P}_{j}\right]=0, \quad\left[\hat{P}_{i}, \hat{G}_{j}\right]=i \delta_{i j} M I} \\
{\left[\hat{G}_{i}, \hat{G}_{j}\right]=0, \quad\left[\hat{H}, \hat{P}_{i}\right]=0,} \\
{\left[\hat{J}_{i}, \hat{J}_{j}\right]=i \varepsilon_{i j k} \hat{J}_{k}, \quad\left[\hat{H}, \hat{G}_{i}\right]=i \hat{P}_{i}} \\
{\left[\hat{J}_{i}, \hat{P}_{j}\right]=i \varepsilon_{i j k} \hat{P}_{k}, \quad\left[\hat{H}, \hat{J}_{i}\right]=0,} \\
{\left[\hat{J}_{i}, \hat{G}_{j}\right]=i \varepsilon_{i j k} \hat{G}_{k}, \quad i, j, k=1,2,3}
\end{gathered}
$$

where $M$ is the mass of the system. All generators of Galilean transformations can be expressed by observables A.1 A.3. We have

$$
\hat{\mathbf{J}}=\hat{\mathbf{S}}+\hat{\mathbf{X}} \times \hat{\mathbf{P}}, \quad \hat{\mathbf{G}}=t \hat{\mathbf{P}}-M \hat{\mathbf{X}}
$$

Moreover in the case of a free particle $\hat{H}=\hat{\mathbf{P}}^{2} / 2 M$.

Irreducible unitary ray representations of the Galilean group are determined by two numbers: the eigenvalue of $\hat{\mathbf{S}}^{2}$, which has the form $s(s+1)$, where $s$ is an integer or a halfinteger and the non-negative real constant $M$. In the sequel we will use the Schrödinger picture. Since $\hat{\mathbf{G}}$ depends on time explicitly, this dependence remains also in Schödinger picture. In momentum representation, we will denote the basis vectors of the carrier space of the determined irreducible unitary representation of the Galilean group (the space of states of the system at time $t$ ) by $|\mathbf{k}, m\rangle_{t}$, where $\mathbf{k}$ is an eigenvalue of momentum operator $\hat{\mathbf{P}}$, and $m$ is the spin component along the $z$ axis. We will denote elements of the unitary representation of the Galilean group as follows:

$$
\begin{aligned}
& U(\mathbf{a})=e^{i \mathbf{a} \cdot \hat{\mathbf{P}}}, \quad U(\mathbf{v})=e^{i \mathbf{v} \cdot \hat{\mathbf{G}}}, \\
& U(R)=e^{i \boldsymbol{\varphi} \cdot \hat{\mathbf{J}}}, \quad U(\tau)=e^{i \tau \hat{H}},
\end{aligned}
$$

where $\mathbf{a}, \mathbf{v}, \boldsymbol{\varphi}$, and $\tau$ are parameters corresponding to pure translations, Galilean boosts, rotations, and time translation, respectively. The action of operators A.12 on basis vectors is the following:

$$
\begin{gathered}
U(\mathbf{a})|\mathbf{k}, m\rangle_{t}=e^{i \mathbf{a} \cdot \mathbf{k}}|\mathbf{k}, m\rangle_{t}, \\
U(R)|\mathbf{k}, m\rangle_{t}=\mathcal{D}^{s}(R)_{m^{\prime} m}\left|R \mathbf{k}, m^{\prime}\right\rangle_{t}, \\
U_{t}(\mathbf{v})|\mathbf{k}, m\rangle_{t}=e^{i t\left[\mathbf{v} \cdot \mathbf{k}-\left(M v^{2} / 2\right)\right]}|\mathbf{k}-M \mathbf{v}, m\rangle_{t},
\end{gathered}
$$

where $\mathcal{D}^{s}(R)$ is the spin $s$ irreducible unitary representation of $\mathrm{SU}(2)$. Moreover we, assume that

$$
e^{i \boldsymbol{\alpha} \cdot \hat{\mathbf{X}}}|\mathbf{k}, m\rangle_{t}=|\mathbf{k}+\boldsymbol{\alpha}, m\rangle_{t} .
$$

The phase factors in Eqs. A.15 and A.16 determine the following form of the vector $|\mathbf{k}, m\rangle_{t+\tau}$ :

$$
|\mathbf{k}, m\rangle_{t+\tau}=e^{-i \tau\left(\mathbf{k}^{2} / 2 M\right)}|\mathbf{k}, m\rangle_{t} .
$$

We denote eigenvectors of the position operator $\hat{\mathbf{X}}$ by $|\mathbf{x}, m\rangle_{t}$. We have

$$
\begin{gathered}
\hat{\mathbf{X}}|\mathbf{x}, m\rangle_{t}=\mathbf{x}|\mathbf{x}, m\rangle_{t} \\
|\mathbf{x}, m\rangle_{t}=\frac{1}{(2 \pi)^{3 / 2}} \int d^{3} \mathbf{k} e^{-i \mathbf{k} \cdot \mathbf{x}}|\mathbf{k}, m\rangle_{t}
\end{gathered}
$$

The Galilean group and operator $e^{i \boldsymbol{\alpha} \cdot \hat{\mathbf{X}}}$ act on vectors $|\mathbf{x}, m\rangle_{t}$ as follows:

$$
\begin{gathered}
U(\mathbf{a})|\mathbf{x}, m\rangle_{t}=|\mathbf{x}-\mathbf{a}, m\rangle_{t}, \\
U(R)|\mathbf{x}, m\rangle_{t}=\mathcal{D}^{s}(R)_{m^{\prime} m}\left|R \mathbf{x}, m^{\prime}\right\rangle_{t}, \\
U_{t}(\mathbf{v})|\mathbf{x}, m\rangle_{t}=e^{i M\left[\left(t \mathbf{v}^{2} / 2\right)-\mathbf{v} \cdot \mathbf{x}\right]}|\mathbf{x}-t \mathbf{v}, m\rangle_{t}, \\
e^{i \boldsymbol{\alpha} \cdot \hat{\mathbf{x}}}|\mathbf{x}, m\rangle_{t}=e^{i \boldsymbol{\alpha} \cdot \mathbf{x}}|\mathbf{x}, m\rangle_{t} .
\end{gathered}
$$

In discussion of EPR-like experiments, it is convinient to use a position basis in which vectors are numbered by spin component along an arbitrary axis (not necessarily the $z$ axis). Thus let $\mathbf{n}$ be an arbitrary unit vector. Observable $\mathbf{n} \cdot \hat{\mathbf{S}}$ measures spin component along an axis in direction $\mathbf{n}$. Since $\mathbf{n} \cdot \hat{\mathbf{S}}$ commutes with $\hat{\mathbf{X}}$, these observables possess a common set of eigenvectors. We denote them by $|\mathbf{x}, \mathbf{n}, \lambda\rangle$,

$$
\begin{gathered}
(\mathbf{n} \cdot \hat{\mathbf{S}})|\mathbf{x}, \mathbf{n}, \lambda\rangle= \\
\left.\hat{\mathbf{X}}|\mathbf{x}, \mathbf{n}, \lambda\rangle=\mathbf{\Sigma})_{\sigma \lambda}|\mathbf{x}, \mathbf{n}, \sigma\rangle=\lambda|\mathbf{x}, \mathbf{x}, \lambda\rangle, \lambda\right\rangle,
\end{gathered}
$$


where values of $\lambda$ are the same as values of $m(\lambda=-s,-s+$ $1, \ldots, s)$ and $\boldsymbol{\Sigma}$ denotes the generators of the representation $\mathcal{D}^{s}$. If we parametrize $\mathbf{n}$ explicitly as follows:

$$
\mathbf{n}=(\sin \theta \cos \varphi, \sin \theta \sin \varphi, \cos \theta),
$$

we get

$$
|\mathbf{x}, \mathbf{n}, \lambda\rangle=\mathcal{D}^{s}\left(e^{i \theta \boldsymbol{\omega} \cdot \boldsymbol{\Sigma}}\right)_{\lambda^{\prime} \lambda}\left|\mathbf{x}, \lambda^{\prime}\right\rangle_{t}
$$

where the vector $\boldsymbol{\omega}$ is orthogonal to $\mathbf{n}$ and

$$
\boldsymbol{\omega}=(\sin \varphi,-\cos \varphi, 0)
$$

We define also the vectors $|\mathbf{k}, \mathbf{n}, \lambda\rangle$ as follows:

$$
|\mathbf{k}, \mathbf{n}, \lambda\rangle=\frac{1}{(2 \pi)^{3 / 2}} \int d^{3} \mathbf{k} e^{i \mathbf{k} \cdot \mathbf{x}}|\mathbf{x}, \mathbf{n}, \lambda\rangle .
$$

One can also check that the following relations hold:

$$
\begin{aligned}
\langle\mathbf{y}, m \mid \mathbf{x}, \mathbf{n}, \lambda\rangle & =\delta(\mathbf{x}-\mathbf{y}) \mathcal{D}^{s}\left(e^{i \theta \boldsymbol{\omega} \cdot \mathbf{\Sigma}}\right)_{m \lambda} \\
\langle\mathbf{p}, m \mid \mathbf{x}, \mathbf{n}, \lambda\rangle & =\frac{e^{-i \mathbf{p} \cdot \mathbf{x}}}{(2 \pi)^{3 / 2}} \mathcal{D}^{s}\left(e^{i \theta \boldsymbol{\omega} \cdot \mathbf{\Sigma}}\right)_{m \lambda}
\end{aligned}
$$

The Galilean group and operator $e^{i \boldsymbol{\alpha} \cdot \hat{\mathbf{X}}}$ act on the vectors $|\mathbf{x}, \mathbf{n}, \lambda\rangle$ as follows:

$$
\begin{gathered}
U(\mathbf{a})|\mathbf{x}, \mathbf{n}, \lambda\rangle=|\mathbf{x}-\mathbf{a}, \mathbf{n}, \lambda\rangle, \\
U(R)|\mathbf{x}, \mathbf{n}, \lambda\rangle=\mathcal{D}^{s}(R)_{\lambda^{\prime} \lambda}\left|R \mathbf{x}, R \mathbf{n}, \lambda^{\prime}\right\rangle, \\
U_{t}(\mathbf{v})|\mathbf{x}, \mathbf{n}, \lambda\rangle=e^{i M\left[\left(t v^{2} / 2\right)-\mathbf{v} \cdot \mathbf{x}\right]}|\mathbf{x}-t \mathbf{v}, \mathbf{n}, \lambda\rangle, \\
e^{i \boldsymbol{\alpha} \cdot \hat{\mathbf{x}}}|\mathbf{x}, \mathbf{n}, \lambda\rangle=e^{i \boldsymbol{\alpha} \cdot \mathbf{x}}|\mathbf{x}, \mathbf{n}, \lambda\rangle .
\end{gathered}
$$

In the case $s=\frac{1}{2}$, we have $\boldsymbol{\Sigma}=\frac{1}{2} \sigma$, so

$$
\begin{aligned}
& \left|\mathbf{x}, \mathbf{n}, \frac{1}{2}\right\rangle=\cos (\theta / 2)\left|\mathbf{x}, \frac{1}{2}\right\rangle+e^{-i \varphi} \sin (\theta / 2)\left|\mathbf{x},-\frac{1}{2}\right\rangle, \\
& \quad\left|\mathbf{x}, \mathbf{n},-\frac{1}{2}\right\rangle=-e^{i \varphi} \sin (\theta / 2)\left|\mathbf{x}, \frac{1}{2}\right\rangle+\cos (\theta / 2)\left|\mathbf{x},-\frac{1}{2}\right\rangle .
\end{aligned}
$$

[1] A. Einstein, B. Podolsky, and N. Rosen, Phys. Rev. 47, 777 (1935).

[2] A. Einstein, in Albert Einstein, Philosopher-Scientist, edited by P. A. Schlipp (Open Court, LaSalle, IL, 1970).

[3] D. Bohm, Quantum Theory (Prentice-Hall, Englewood Clifs, 1951).

[4] J. S. Bell, Physics (Long Island City, N.Y.) 1, 195 (1964).

[5] A. Aspect, P. Grangier, and G. Roger, Phys. Rev. Lett. 47, 460 (1981).

[6] A. Aspect, P. Grangier, and G. Roger, Phys. Rev. Lett. 49, 91 (1982).

[7] W. Tittel et al., Phys. Rev. A 57, 3229 (1998).

[8] W. Tittel, J. Brendel, H. Zbinden, and N. Gisin, Phys. Rev. Lett. 81, 3563 (1998).

[9] G. Weihs et al., Phys. Rev. Lett. 81, 5039 (1998).

[10] A. Apostolakis et al., Phys. Lett. B 422, 339 (1998).

[11] R. Foadi and F. Selleri, Phys. Lett. B 461, 123 (1999).

[12] H. Zbinden, J. Brendel, N. Gisin, and W. Tittel, Phys. Rev. A 63, 022111 (2001).

[13] L. E. Ballentine, Quantum Mechanics: A Modern Development
(World Scientific, Singapore, 1998).

[14] A. Peres, Quantum Theory: Concepts and Methods (Kluwer, Dordrecht, 1995).

[15] I. V. Volovich, e-print quant-ph/0012010

[16] I. V. Volovich, e-print quant-ph/0108073

[17] H. Bacry, Localizability and Space in Quantum Physics, Lecture Notes in Physics Vol. 308 (Springer-Verlag, Berlin, 1988).

[18] M. Czachor, Phys. Rev. A 55, 72 (1997).

[19] M. Czachor, in Photonic Quantum Computing, edited by S. P. Hotaling and A. R. Pirich [Proc. SPIE 3076, 141 (1997)].

[20] P. Caban and J. Rembieliński, Phys. Rev. A 59, 4187 (1999).

[21] J. Rembieliński and K. A. Smoliński, Phys. Rev. A 66, 052114 (2002).

[22] N. Gisin and H. Zbinden, Phys. Lett. A 246, 103 (1999).

[23] For technical reasons, there may exist some loopholes (see, e.g., [22] and references therein) that allow us to maintain the local and realistic interpretation of quantum mechanics, but we will not consider them here. 